\title{
The Clar Numbers of Capped Nanotubes
}

\author{
Jack E. Graver ${ }^{a}$, Elizabeth J. Hartung ${ }^{b}$ \\ ${ }^{a}$ Syracuse University, Syracuse, NY, USA \\ ${ }^{b}$ Massachusetts College of Liberal Arts, MA, USA \\ jegraver@syr.edu, e.hartung@mcla.edu
}

(Received September 2, 2021)

\begin{abstract}
A nanotube is a closed carbon molecule in the shape of a capped cylinder. The Clar number of a carbon molecule is the maximum number of independent benzene rings over all possible Kekulé structures. We prove that at most two Clar chains are required on nanotube cylinders, giving lower bounds on the Clar number of nanotubes. In other words, a fully conjugated $\pi$-system running along the nanotube's cylinder will be broken by at most two fracture lines. In [8], this double bond structure of capped nanotubes was described, but without a detailed mathematical proof that at most two Clar chains are required across a nanotube cylinder. We use this result to settle a conjecture in the case of long nanotubes. Carr, Wang and Ye proved that the Clar number for fullerenes on $v$ vertices is bounded below by $\frac{v-380}{61}$ and further conjectured that the sharp lower bound is $\frac{v-20}{10}[4]$. We prove that this sharp lower bound holds for nanotubes of sufficient length. We also give a formula for the maximum number of vertices in the cap of a nanotube with chiral indices $(n, m)$, where the caps are defined as in [3].
\end{abstract}

\section{Introduction}

Fullerenes model closed carbon molecules. A fullerene is a 3-regular plane graph whose faces are hexagons and pentagons, and it can be shown using Euler's formula that any fullerene has exactly 12 pentagons. A nanotube is a fullerene in the shape of a capped cylinder. The cylinder is made up entirely of hexagons, and each capped end contains six pentagons and some number of hexagons. Carbon nanotubes have many desirable physical and electrical properties, and are widely used in a variety of applications $[1,6,14,15]$. Nanotubes can have extremely large length-to-width ratios; nanotubes with a length-towidth ratio of 132,000,000:1 have been grown [20]. 
The cylinder of a nanotube can be described by cutting out a rectangle in the hexagonal tessellation and gluing its sides together. The angle and shape of the cylinder are given by its chiral indices, $(n, m)$. The chiral indices are the coordinates of a vector with basis vectors in the hexagonal tessellation, as shown in on the left of Figure 1. It is assumed that $n \geq m$ to avoid mirror images. A nanotube with chiral indices $(7,3)$ is shown on the right in Figure 1. We use this same coordinate system to describe the distance between faces in the nanotube cylinder.

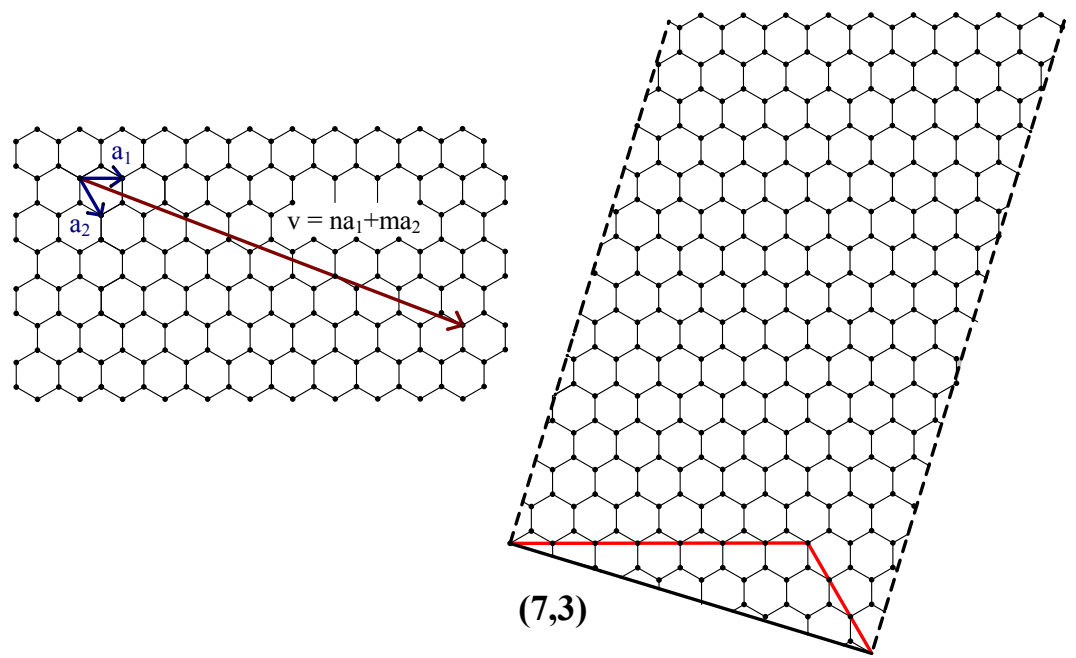

Figure 1. The chiral indices $(n, m)$ describe the shape of a nanotube cylinder. Left: vector for chiral indices. Right: $(7,3)$ nanotube. The dashed edges along the sides are identified to create a cylinder.

Each carbon atom of a fullerene is incident with exactly one double bond, so the edges of the double bond structure form a perfect matching of the graph. In the literature, perfect matchings in fullerenes and chemical graphs are often called Kekulé structures. By Petersen's Theorem [16], a fullerene will always have at least one Kekulé structure, and in fact, a fullerene has exponentially many Kekulé structures in terms of its number of vertices [13].

Given a Kekulé structure for a fullerene, a benzene ring is a hexagon with three of its double bonds in the Kekulé structure. The Clar number of a fullerene $G$ is the maximum number of independent benzene rings over all possible Kekulé structures for $G$. The Clar number was introduced in [5] and is related to the electronic and magnetic properties of 
various carbon structures $[2,7,18,19,21]$.

Let $C$ be a maximal independent set of benzene rings in a Kekulé structure $K$, and let $A$ be the set of edges of $K$ that do not lie on any benzene ring. Then each vertex of $G$ is incident with exactly one element from $C \cup A$, so $(C, A)$ forms a vertex covering of $G$. Such a face-edge vertex covering of a fullerene is called a Clar structure, and was introduced in [10] and further described in [9], [12]. The Clar number of a fullerene on $v$ vertices is given by $|C|=\frac{v}{6}-\frac{|A|}{3}$, and therefore, finding a Kekulé structure that minimizes the number of edges in $A$ is equivalent to finding the Clar number of a fullerene.

The edges of $A$ form six Clar chains connecting pairs of pentagons over a fullerene [12]. These are alternating sequences of faces of the fullerene and edges of $A$ (see Fig 2). Properties of Clar chains and details of when two pentagons can be paired by a Clar chain are given in [9]. The number of edges of $A$ in a Clar chain between two pentagons is linearly related to the distance between those pentagons. Thus, in a Clar structure that gives the Clar number, the pentagons paired by Clar chains are typically "close" to one another.
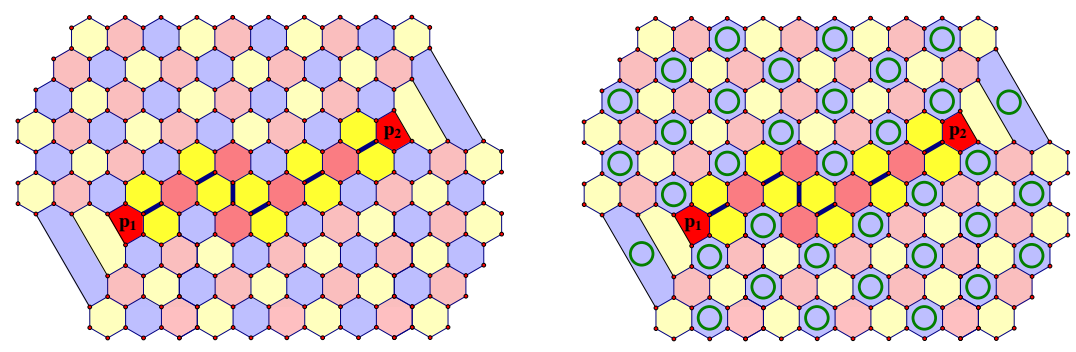

Figure 2. Left: A Clar chain pairing two pentagons, $p_{1}$ and $p_{2}$. The edges of the chain are shown as thick blue edges. This is a red-yellow chain: the pentagons and faces of the chain are red; the augmenting faces are yellow. Right: Outside of the Clar chain, all blue faces are independent benzene rings, indicated by green circles.

Given a Clar structure $(C, A)$ for a fullerene, the fullerene admits a partial face 3coloring in which the improperly colored faces are exactly those that have a bounding edge in $A$ [9]. We call the faces with bounding edges in $A$ the augmenting faces of the Clar chains. The faces of a Clar chain are all in one color class, and the augmenting faces are in a second color class (see Fig. 2). Given a Clar structure $(C, A)$ and its associated improper face 3-coloring, we describe a chain by these two colors. For example, if the faces of the chain are red and its augmenting faces are blue, we call the chain a red-blue 
chain. Figure 2 shows a red-yellow Clar chain. The six chains giving a Clar structure have the property that they involve just two colors, e.g. red-blue and blue-red chains. Outside of the chains, the faces in the remaining color class (e.g. yellow) are independent benzene rings of this Clar structure.

The extreme length-to-width ratios of nanotubes make the pairing of pentagons by Clar chains particularly interesting. If the six pentagons in each cap can be paired among themselves, then the Clar chains will occur within the caps and will be relatively short, so the Clar number of the nanotube will be large. If two pentagons on opposite caps must be paired, then there will be Clar chains running across the length of the cylinder, decreasing the Clar number.

In [8], the authors showed that when the chiral indices $(n, m)$ of a nanotube are congruent mod 3 , the pentagons within each cap can be paired, and thus there are no edges of $A$ over the cylinder. When the chiral indices are not congruent mod 3, at least two Clar chains must run across the cylinder, connecting two pairs of pentagons in opposite caps. Figure 3 shows the Clar structure for cylinders with chiral indices $(7,3),(7,4)$, and $(7,5)$. Here, the coloring associated with the Clar chains is included. Figure 4 shows nanotubes where all pentagons are paired by Clar chains.

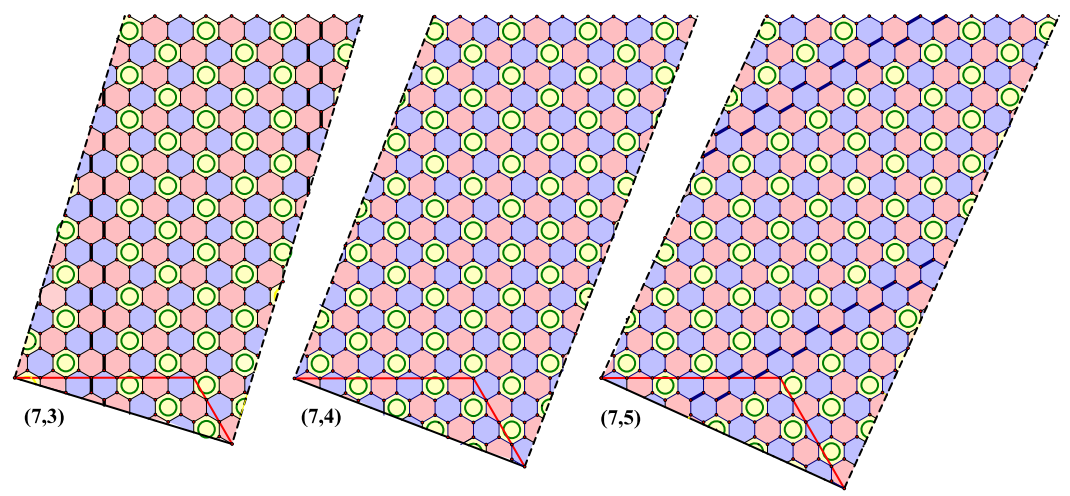

Figure 3. The Clar structure for nanotubes with chiral indices $(7,3),(7,4)$, and $(7,5)$, respectively. Here, the two chains are shown adjacent to one another. Green circles show independent benzene rings, dark blue edges are edges of the Clar chains. 

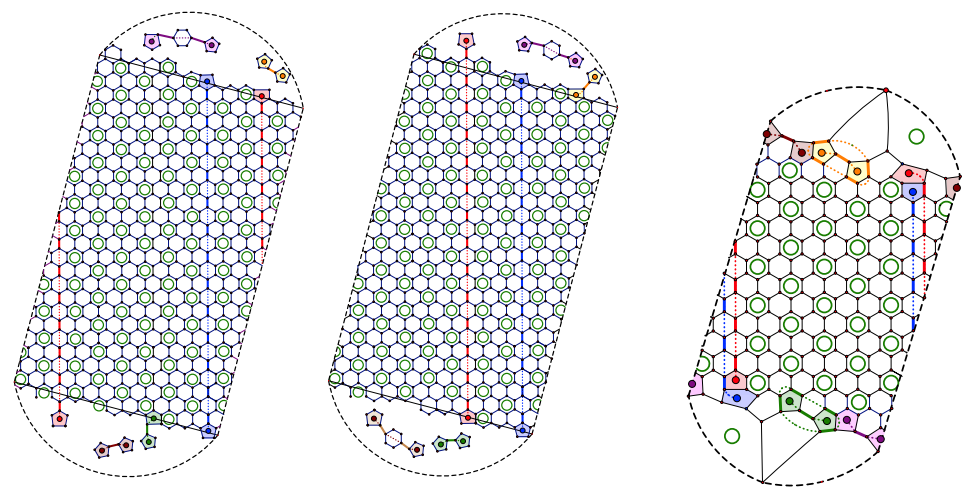

Figure 4. Pentagon pairings over nanotubes with chiral indices not congruent mod 3. There are two fracture lines of double bonds across the cylinder. The left and center image show an $(11,4)$ nanotube without specific caps. These two images show different pairings of pentagons, but both result in two fracture lines, one red pair and one blue pair. Note that, if the crossing chains were the same color, the initial pentagons could be paired within the cap. On the right, a $(7,3)$ nanotube is shown with a specific cap on the top and bottom, and a completed Clar structure. Here, the Clar chains across the cylinder are adjacent.

The proof that at most two chains would be required was left for a more mathematically detailed paper, as [8] was intended for a broader audience. In the next section, we write up the details of this proof. In Section 3, we describe the caps in more detail and give a formula for the maximum number of vertices in a nanotube cap with chiral indices $(n, m)$. In Section 4, we show that a conjecture on the minimum Clar number of a fullerene holds for nanotubes of sufficient length.

\section{Proof of two chains theorem}

We wish to show that only two Clar chains are required across the cylinder of a nanotube with coordinates $(n, m)$ when $n-m \not_{3} 0$. We know that when $n-m \equiv_{3} 0$, no chains across the cylinder are required [8]. We will show that when $n$ and $m$ are not congruent mod 3, the Kekulé structure for the cylinder given in Figure 3 can be extended to the nanotube caps. We start by defining a partitioning rim around the cylinder.

Suppose that the two chains crossing the cylinder are adjacent and that the first chain is a red-blue chain (see Fig 5). Because the chains are adjacent, the blue augmenting faces 
on one side of the red-blue chain are also the faces of the second chain. The red faces of the red-blue chain are augmenting faces on the second chain. So the second chain is a blue-red chain. Along these chains, there are two adjacent red faces. Over the cylinder, the faces of the third color class, the yellow faces, are independent benzene rings. The Kekulé edges on these independent benzene rings can exit red or blue faces; select the rotation so that they exit red faces.
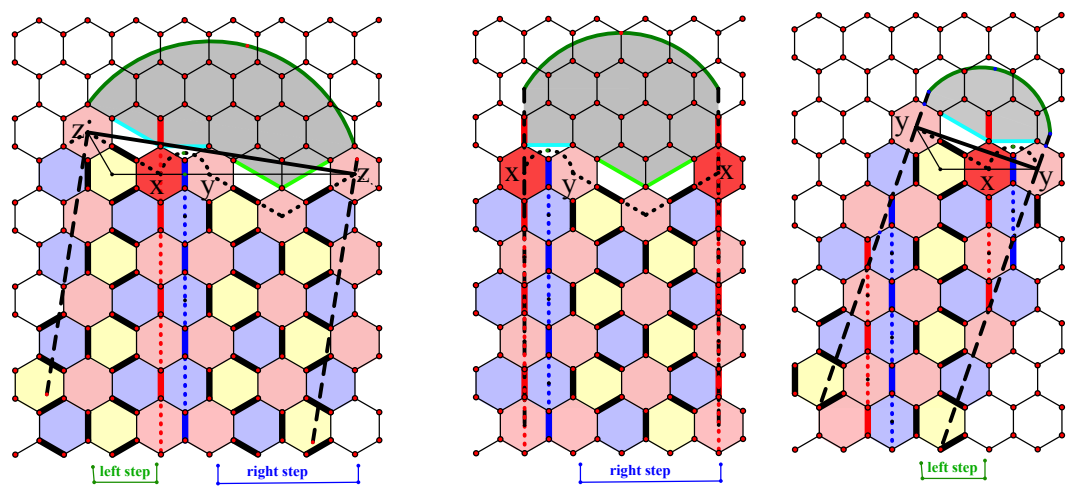

Figure 5. Partitioning rims when $n-m \equiv_{3} 1$. The partitioning rim is shown as a dotted line going around the circumference of the cylinder.

Consider the case that $n-m \equiv_{3} 1$. Label the two adjacent red faces at the top of the chain $x$ and $y$ as shown in Figure 5. The partitioning rim goes around the circumference of the cylinder, beginning and ending at the center of $x$. Starting at the center of $x$, the partitioning rim goes over the blue-red chain to the center of $y$, and then makes some number of right steps and left steps to end back at the center of $x$. On the left in Figure 5 , the dotted edges going from the center of $y$ to the center of $z$ is a right step, the strip that is one and a half faces wide and goes from $z$ to $x$ is a left step. We see that a right step will have coordinates $(3,0)$ and a left step will have coordinates $(1,1)$.

Lemma 1 Given a nanotube with coordinates $(n, m)$ where $n-m \equiv_{3} 1$, assume that the cylinder admits a Kekulé structure which is perfect except for two adjacent chains. Then a partitioning rim exists as described above.

Proof. The smallest cylinders such that $n-m \equiv_{3} 1$ are shown with their partitioning rims in the center and right images of Figure 5, a $(4,0)$ nanotube, whose partitioning rim has no left steps, and a $(2,1)$ nanotube, whose partitioning rim has no right steps. Observe 
that neither of these cylinders is wide enough to have a cap. However, we use these as a base for construction of all cylinders with chiral indices in this modularity class. A left step has coordinates $(1,1)$ and a right step has coordinates $(3,0)$. We see that any $(n, m)$ nanotube with $n-m \equiv_{3} 1$ can be obtained by starting with a $(4,0)$ nanotube or a $(2,1)$ nanotube and adding some number of left and right steps. Specifically, if $n-m \equiv_{3} 1$, then $n=3 k+m+1$, where there are $k$ right steps and $m$ left steps. Thus a partitioning rim can be constructed for any nanotube with $n-m_{3} \equiv 1$.

When $n-m=2$, the only difference in the partitioning rim is the behavior above the two adjacent chains (see Figure 6). As before, label the two adjacent red faces at the top of the chain $x$ and $y$. Because of the angle of the chains when $n-m=2$, we also label the red face $w$ in the second chain above $y$. Starting at the center of $x$, the partitioning rim goes over the blue-red chain to the next vertex of $y$ clockwise. It then continues to the center of $w$. The partitioning rim then makes some number of left and right steps to end back at $x$.
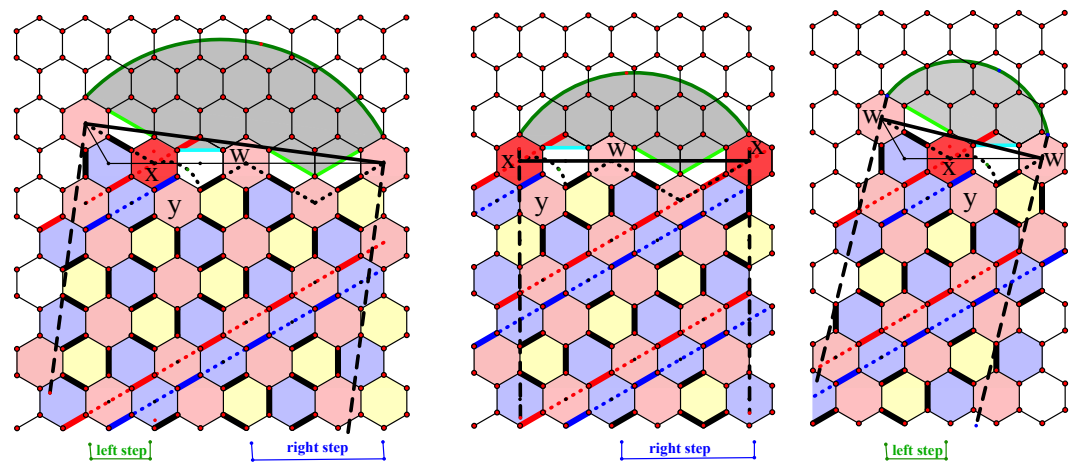

Figure 6. Partitioning rims when $n-m \equiv_{3} 2$. The partitioning rim is shown as a dotted line going around the circumference of the cylinder.

Lemma 2 Given a nanotube with coordinates $(n, m)$ where $n-m \equiv_{3} 2$, assume that the cylinder admits a Kekulé structure which is perfect except for two adjacent chains. Then a partitioning rim exists as described above.

Proof. The smallest cylinders such that $n-m \equiv_{3} 2$ are shown with their partitioning rims on the center and right of Figure 6, a $(5,0)$ nanotube, whose partitioning rim has no left steps, and a $(3,1)$ nanotube, whose partitioning rim has no right steps. Note that 
the $(3,1)$ cylinder again is not wide enough to accommodate a nanocap, but we use this as a base case. Any $(n, m)$ nanotube with $n-m \equiv_{3} 2$ can be obtained by starting with a $(5,0)$ nanotube or a $(3,1)$ nanotube and adding some number of left and right steps. Specifically, if $n-m \equiv_{3} 2$, then $n=3 k+m+1$ where there are $k$ right steps and $m$ left steps. Thus a partitioning rim can be constructed for any nanotube with $n-m \equiv_{3} 2$.
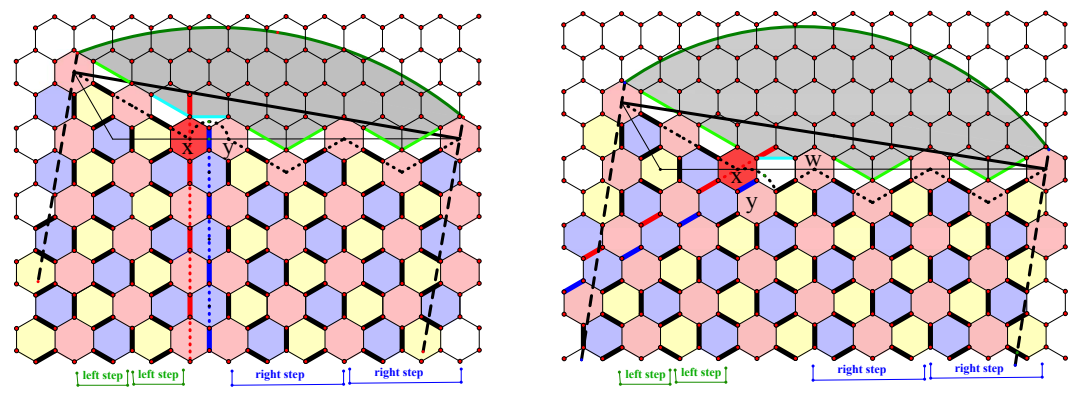

Figure 7. Partitioning rims for cylinders $(9,2)$ and $(10,2)$

Now that we have defined the partitioning rim and checked that it exists for all cylinders with $n-m \not_{3} 0$, we use it to extend the Kekulé structure of the cylinder into the caps.

Theorem 1 Let $G$ be a nanotube whose cylinder admits a Kekulé structure which is perfect except for two adjacent chains as illustrated in Figure 1. Then G admits a full Kekulé structure that incorporates the cylinder's Kekulé structure.

Proof. We assume that the cylinder chains are a red-blue chain and an adjacent blue-red chain. Outside of the chains, we select a rotation of the Kekule edges so that they exit red faces. The yellow faces are the independent benzene rings of a Kekulé structure for the cylinder.

Construct the partitioning rim for the cylinder as described above and pictured in Figure 7 . We partition the edges and vertices of the nanotube in the following way: edges and vertices that are incident with the partitioning rim or lie below it (actually, on or between the top and bottom rims) belong to the cylinder. The vertices and edges that lie above this rim belong to the top cap; those that lie below the bottom rim belong to the bottom cap. The vertices in the cylinder are all paired by the cylinder's Kekulé structure. 
Observe that the top edge of the red chain lies entirely in the cap while the top edge of the blue chain lies entirely in the cylinder.

We wish to construct a Kekulé structure for the cap; however, the plane graph of this cap has some vertices of degree 1 and 2 on its boundary. We construct an extended cap by adding the green and light blue edges as illustrated in the figures. Specifically, we add a green edge above each left step, two adjacent green edges above each right step, and one or two light blue edge above the chains. When $n-m \equiv_{3} 1$, two light blue edges are required, each incident with the bottom vertex of the top edge of the red chain. When $n-m \equiv_{3} 2$, one light blue edge is added and is also incident with the bottom vertex of the top edge of the red chain.

This extended cap is now 3-regular and of even order. Plesnik showed that a $(k-1)$ edge connected $k$-regular graph of even order has a perfect matching that includes any given edge [17]. Thus, the extended cap has a perfect matching that includes the red edge in the cap and therefore excludes the light blue edge(s) from the matching. Now reattach this extended cap to the cylinder and we have a perfect matching for the cylinder plus the extended cap. However, the added light blue and green edges do not belong to the fullerene and must be deleted. As noted above, the light blue edge(s) are not part of the matching and therefore can be deleted as can every green edge is not in the matching. Finally, consider any green edge that is part of the matching. It lies on a quadrilateral opposite a Kekulé edge of the partitioning rim. Simply replace the green edge and opposite Kekulé edge by the other two edges of the quadrilateral and delete the green edge.

Corollary 1 The chain decomposition of a nanotube will never require more than two chains running through the cylinder.

Proof. By Theorem 1, there is a perfect matching for the nanotube that includes the edges of the adjacent Clar chains. Because these are Clar chains, they terminate in pentagons in each cap. Since the cylinder outside of these adjacent chains can be 3-colored, there are no other chains across the length of the cylinder, and the remaining four pentagons in each cap can be paired in that cap.

Finally, consider all chain decompositions for the nanotube and observe that, since a nanotube cylinder is "very long," a decomposition involving more than two chains running the length of the cylinder cannot be a shortest chain decomposition. However we note 
that the actual shortest chain decomposition will involve two parallel, but not necessarily adjacent, chains running the length of the cylinder.

\section{$3 \quad$ Nanotube caps}

A nanotube consists of two caps separated by a cylinder composed entirely of hexagons. Each cap contains exactly six pentagons and some number of hexagons. To accommodate the six pentagons, a cap can have chiral indices $(5,0)$ or $(n, m)$ where $n+m \geq 6$. To define the caps more precisely, we follow [3]. For a nanotube with chiral indices $(n, m)$, the cap can always be chosen with the property that at least one pentagon is on the boundary of the cap, and the degree sequence of the vertices on the cap boundary is of the form $(23)^{n}(32)^{m}$. There is a unique cap with chiral indices $(5,0)$, but for $n+m \geq 6$, there are many possible caps of this form, and the number of different caps grows as $n$ and $m$ get larger [3]. Figure 8 shows two caps for each of the chiral indices $(8,0)$ and $(5,3)$, respectively. The bottom two caps are have the maximum number of vertices for these chiral indices.

$(23)^{8}$
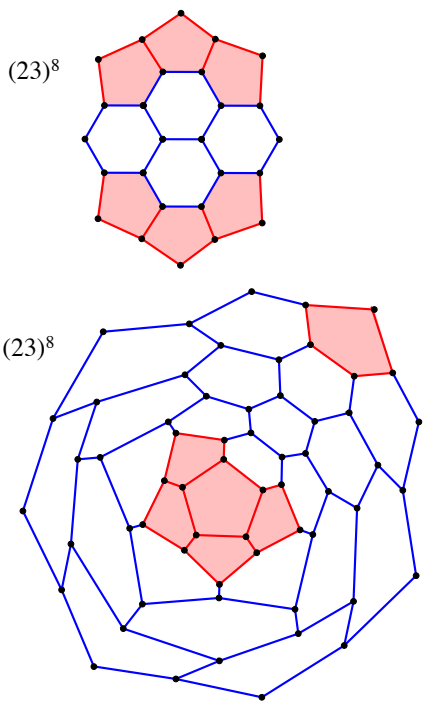

$(23)^{5}(32)^{3}$

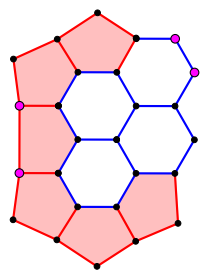

$(23)$

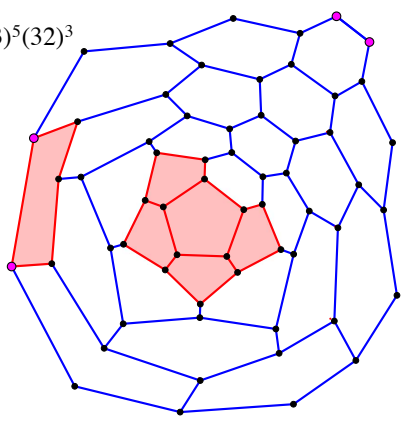

Figure 8. Different caps for chiral indices $(8,0)$ (left) and $(5,3)$ (right). In the $(5,3)$ caps, the adjacent degree 2 and degree 3 boundary vertices are pink. The bottom two caps are have the maximum number of vertices for these indices. 
Among all possible caps for chiral indices $(n, m)$, the cap with the largest number of vertices is attained by arranging five pentagons in a spiral, then continuing with hexagons and placing the sixth pentagon to give the desired chiral indices [3]. Begin with a spiral of 5 pentagons, and complete the cycle of faces with a hexagon. We label this hexagon as being in position 1. This layer has five faces; each subsequent cycle will be one face longer than the previous. The faces of each layer are numbered such that the face adjacent with hexagon 1 in the previous layer is also labeled 1, and the faces are then numbered clockwise. To create a cap of maximum size with chiral indices $(n, m)$, place the sixth pentagon in the $(n+1)$ th position of the length $n+m$ cycle. In the case of chiral indices $(n, 0)$, the sixth pentagon will be placed in the first position of the length $n$ cycle. See Figure 9 .

$(5,0)$

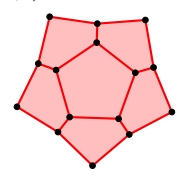

$(23)^{5}$

$(23)^{4}(32)^{3}$

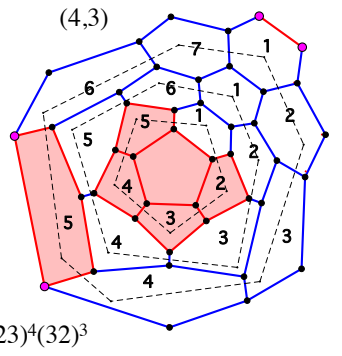

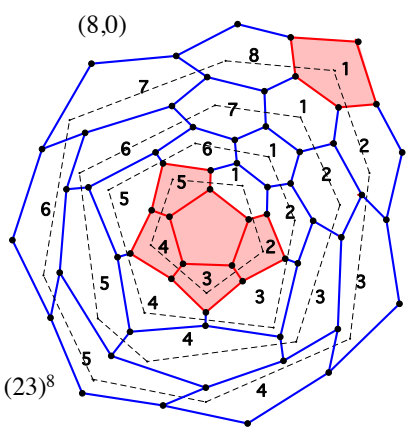

Figure 9. Left: unique $(5,0)$ cap. Center and right: Construction of caps with maximum vertices for $(4,3)$ and $(8,0)$, respectively. A maximum size $(n, m)$ cap is created by placing the sixth pentagon in the $(n+1)$ th position of the length $n+m$ cycle. In the case of chiral indices $(n, 0)$, the sixth pentagon will be placed in position 1 of the length $n$ cycle.

Lemma 3 The maximum number of vertices in a nanocap with chiral indices $(n, m)$ is $(n+m)^{2}+2(n+m)-20$.

Proof. Let $n+m=k$. There are 5 vertices around the central pentagon. The next layer of faces has length 5 and each subsequent layer has one additional face. A layer with $j$ faces adds $2 j+1$ vertices, except the last layer, which has $2 j$ vertices because of the pentagon on the boundary. Thus the total number of vertices in the cap is 


$$
\begin{aligned}
& 5+\sum_{j=5}^{k-1}(2 j+1)+2 k=5+2 \sum_{j=5}^{k-1} j+\sum_{j=5}^{k-1} 1+2 k \\
& =5+k(k-1)-2(1+2+3+4)+(k-1-4)+2 k \\
& =5+k^{2}-k-20+k-5+2 k=k^{2}+2 k-20
\end{aligned}
$$

\section{Nanotubes and the Clar number}

A sharp upper bound for the Clar number of a fullerene on $v$ vertices is $\frac{v}{6}-2$, as was shown in [23], and fullerenes that attain this bound were characterized in [11], [22]. Recall that the Clar number of a fullerene $G$ on $v$ vertices is given by $C(G)=\frac{v}{6}-\frac{|A|}{3}$, where $|A|$ is the number of edges in the Clar chains. Fullerenes attain the upper bound $\frac{v}{6}-2$ when $|A|=6$, that is, the 12 pentagons of the fullerene can be paired such that each of the six Clar chains between pentagons consists of a single edge of $A$.

The fact that at most two Clar chains are required over a nanotube cylinder enables us to prove that a conjecture for the minimum Clar number for fullerenes holds for sufficiently long nanotubes. In [4], Carr, Wang, and Ye proved that the Clar number of a fullerene on $v$ vertices is bounded below by $\frac{v-380}{61}$. They further conjectured the following:

Conjecture 2.6 [4]: Let $\mathrm{G}$ be a fullerene graph with $v$ vertices. Then $C(G) \geq \frac{v-20}{10}$ and equality holds if and only if $\mathrm{G}$ contains a non-trivial cyclic 5-edge-cut.

We define the length of a nanotube to be the number of rows of vertices over the cylinder, not including the vertices on the cap boundaries. Each row of the cylinder in an $(n, m)$ nanotube has $2(n+m)$ vertices, so there are $2 L(n+m)$ vertices over the cylinder.

Our construction of the Kekulé structure for the extended cap in Theorem 1 has the property that some of the edges of the Kekule structure in the row of vertices immediately below each cap can be disrupted. This occurs if a green edge of the construction is in the Kekulé structure and must be swapped for the other bounding edges of its hexagon. Therefore, we remove the next row of vertices from each end of the cylinder in considering the contribution to the Clar number from the cylinder. Thus, $V_{c}=2(L-2)(n+m)$ is a lower bound for the number of vertices on the cylinder over which the described Kekulé structure is guaranteed.

The number of edges of $|A|$ contained in the Clar chains running across the cylinder for each congruence class of $n-m$ modulo 3 is given on page 1090 of [8]. This result, 
together with the above discussion on the length of a nanotube cylinder, can be stated as the following lemma and Theorem:

Lemma 4 Let $G$ be a nanotube with chiral indices $(n, m)$ and length $L$. Let $V_{c}=2(L-$ $2)(n+m)$. Then the number of edges of $A$ over the cylinder is

1. 0 when $n-m \equiv_{3} 0$

2. $\frac{V_{c}}{3(2 n+m)}$ when $n-m \equiv_{3} 1$

3. $\frac{V_{c}}{3(n+2 m)}$ when $n-m \equiv_{3} 2$

Theorem 2 Let $G$ be a nanotube with chiral indices $(n, m)$ and length $L$. Let $V_{c}=$ $2(L-2)(n+m)$. Then the contribution from the cylinder to the Clar number of $G$ is at least:

1. $\frac{V_{c}}{6}$ when $n-m \equiv_{3} 0$

2. $\frac{V_{c}}{6}-\frac{V_{c}}{3(2 n+m)}$ when $n-m \equiv_{3} 1$

3. $\frac{V_{c}}{6}-\frac{V_{c}}{3(n+2 m)}$ when $n-m \equiv_{3} 2$

Using Theorem 2, we can show that the contribution to the Clar number from the nanotube cylinder alone achieves the bound of the conjecture.

Theorem 3 For any pair of chiral indices $(n, m)$, there is a length $L(n, m)$ such that any nanotube $G$ with chiral indices $(n, m)$ and length at least $L(n, m)$ satisfies $C(G) \geq \frac{v-20}{10}$. In particular,

1. If $n-m \equiv_{3} 0$, then $L(n, m)=\frac{3(n+m+2)}{2}-\frac{45}{n+m}+5$

2. If $n-m \equiv_{3} 1$, then $L(n, m)=\frac{n+m+2}{10(R-.1)}-\frac{3}{(n+m)(R-.1)}+\frac{2 R}{R-.1}$, where $R=\frac{1}{6}-\frac{1}{3(2 n+m)}$

3. If $n-m \equiv_{3} 2$, then $L(n, m)=\frac{n+m+2}{10(R-.1)}-\frac{3}{(n+m)(R-.1)}+\frac{2 R}{R-.1}$ where $R=\frac{1}{6}-\frac{1}{3(n+2 m)}$

Proof. Theorem 2 gives the contribution to the Clar number over the cylinder for each congruence class of $n-m$ modulo 3 . Let $R$ be the ratio by which $V_{c}$ is multiplied in Theorem 2. So when $n-m \equiv_{3} 0, R=\frac{1}{6}$; when $n-m \equiv_{3} 1, R=\frac{1}{6}-\frac{1}{3(2 n+m)}$; when $n-m \equiv_{3} 2, R=\frac{1}{6}-\frac{1}{3(n+2 m)}$. 
From Lemma 3, the maximum number of vertices in each cap is $(n+m)^{2}+2(n+m)-20$, and so the maximum number of vertices in the two caps together is $M=2\left((n+m)^{2}+\right.$ $2(n+m)-20)$. The maximum number of vertices in $G$ is then the number of vertices in the cylinder plus $M$. That is, $v \leq 2 L(n+m)+M=2 L(n+m)+2\left((n+m)^{2}+2(n+m)-20\right)$.

We want to show that contribution to the Clar number from the nanotube cylinder is at least $\frac{v-20}{10}$ for nanotubes of sufficient length. Thus, we want to find the bound on $L$ such that

$R \cdot\left|V_{c}\right| \geq \frac{v-20}{10}$. Substituting our maximum value for $v$ and minimum for $\left|V_{c}\right|$ gives: $2 R(L-2)(n+m) \geq \frac{2 L(n+m)+2\left((n+m)^{2}+2(n+m)-20\right)-20}{10}$. Solving for $L$ gives

$$
L \geq \frac{n+m+2}{10(R-.1)}-\frac{3}{(n+m)(R-.1)}+\frac{2 R}{R-.1} .
$$

Observe that in the case $n-m \equiv_{3} 0, R=\frac{1}{6}$, and this simply reduces to $L \geq$ $\frac{3(n+m+2)}{2}-\frac{45}{n+m}+5$.

Table 1 Shows the length bound, $L(m, n)$, for each pair of chiral indices $(n, m)$ with $m \leq n \leq 12$. $L(5,0)$ is 0 , because Carr, Wang and Ye proved that the Clar number of a fullerene with a nontrivial cyclic 5-edge-cut is $\frac{v-20}{6}[4]$, and these correspond exactly with $(5,0)$ nanotubes (including the dodecahedron, which has length $L=0)$. The other values of the table are a direct application of the formulas from Theorem 3 , where each value is rounded up to the nearest whole number. The cells of the table are colored to highlight the congruence classes modulo 3 of $n-m$.

Table 1. Entries of the table represent $L(n, m)$, the length such that any nanotube $G$ with chiral indices $(n, m)$ and length at least $L(m, n)$ is guaranteed to satisfy $C(G) \geq \frac{v-20}{10}$.

\begin{tabular}{|c|c|c|c|c|c|c|c|c|c|c|c|c|c|}
\hline $\mathrm{n}$ & 0 & 1 & 2 & 3 & 4 & 5 & 6 & 7 & 8 & 9 & 10 & 11 & 12 \\
\hline 3 & & & & 10 & & & & & & & & & \\
\hline 4 & & & 23 & 21 & 15 & & & & & & & & \\
\hline 5 & 0 & 16 & 13 & 25 & 25 & 19 & & & & & & & \\
\hline 6 & 10 & 29 & 22 & 17 & 28 & 29 & 23 & & & & & & \\
\hline 7 & 18 & 15 & 29 & 26 & 21 & 31 & 32 & 26 & & & & & \\
\hline 8 & 36 & 23 & 19 & 31 & 30 & 25 & 34 & 35 & 30 & & & & \\
\hline 9 & 17 & 33 & 27 & 23 & 34 & 33 & 28 & 37 & 38 & 33 & & & \\
\hline 10 & 25 & 21 & 34 & 31 & 26 & 37 & 36 & 31 & 40 & 41 & 36 & & \\
\hline 11 & 36 & 28 & 25 & 36 & 34 & 30 & 39 & 39 & 35 & 43 & 44 & 39 & \\
\hline 12 & 23 & 37 & 32 & 28 & 39 & 37 & 33 & 42 & 43 & 38 & 46 & 47 & 43 \\
\hline
\end{tabular}


The bound on the length, $L(n, m)$, assumes a "worst case scenario" at multiple stages. Importantly, we assume no contribution to the Clar number from the caps. Especially as $n$ and $m$ increase, Clar faces on the caps are expected. We also assume that the first row of vertices below each cap do not contribute to the Clar number. Finally, we assume that we have the largest caps possible for chiral indices $(n, m)$, resulting in the worst possible comparison for the Clar contribution from the cylinder versus the lower bound for the entire nanotube. Thus, the length requirement for nanotubes to satisfy the conjecture could very likely be improved, or even eliminated, with methods such as a better understanding of the Clar chains and contribution to the Clar number from the caps.

\section{References}

[1] P. Avouris, M. Freitag, V. Perebeinos, Carbon-nanotube photonics and optoelectronics, Nature Photon. 2 (2008) 341-350.

[2] D. Bhattacharya, A. Panda, A. Misra, D. Klein, Clar theory extended for polyacenes and beyond, J. Phys. Chem. A. 118 (2014) 4325-4338.

[3] G. Brinkmann, U. v. Nathusius, A. H. R. Palser, A constructive enumeration of nanotube caps, Discr. Appl. Math. 116 (2002) 55-71.

[4] J. A. Carr, X. Wang, D. Ye, Packing resonant hexagons in fullerenes, Discr. Opt. 13 (2014) 49-54.

[5] E. Clar, The Aromatic Sextet, Wiley, London. 1972.

[6] M. F. L. De Volder, S. H. Tawfick, R. H. Baughman, A. J. Hart, Carbon nanotubes: present and future commercial applications, Science 339 (2013) 535-539.

[7] T. Enoki, Role of edges in the electronic and magnetic structures of nanographene, Phys. Scr. 2012 (2012) \#014008.

[8] J. E. Graver, E. J. Hartung, A. Williams, Resonance structures and aromaticity in capped carbon nanotubes, Carbon 173 (2021) 1082-1092.

[9] J. E. Graver, E. J. Hartung, The Clar and Fries structures of a fullerene, Discr. Appl. Math. 215 (2016) 112-125.

[10] E. J. Hartung, The Clar Structure of Fullerenes, Ph.D. dissertation. Syracuse Univ., 2012. 
[11] E. J. Hartung, Fullerenes with complete Clar structure, Discr. Appl. Math. 161 (2013) 2952-2957.

[12] E. J. Hartung, Clar chains and a counterexample, J. Math. Chem. 52 (2014) 9901006.

[13] F. Kardoš, D. Král', J. Miškuf, J. Sereni, Fullerene graphs have exponentially many perfect matchings, J. Math. Chem. 46 (2009) 443-447.

[14] S. N. Kim, J. F. Rusling, F. Papadimitrakopoulos, Carbon nanotubes for electronic and electrochemical detection of biomolecules, Adv. Mat. 19 (2007) 3214-3228.

[15] W. Lu, C. M. Lieber, Nanoelectronics from the bottom up, Nature Mat. 6 (2007) 841-850.

[16] J. Petersen, Die Theorie der Regulären Graphs, Acta Math. 15 (1891) 193-220.

[17] J. Plesnik, Connectivity of regular graphs and the existence of 1-factors. Mat. Cas. Slov. Akad. Vied. 22 (1972) 310-318.

[18] G. Portella, J. Poater, M. Sola, Assessment of Clar's aromatic $\pi$-sextet rule by means of PDI, NICS, and HOMA indicators of local aromaticity, J. Phys. Org. Chem. 18 (2005) 785-791.

[19] M. Randić, Aromaticity of polycyclic conjugated hydrocarbons, Chem Rev. 103 (2003) 3449-3606.

[20] X. Wang, Q. Li, J. Xie, Z. Jin, J. Wang, Y. Li, K. Jiang, S. Fan, Fabrication of ultralong and electrically uniform single-walled carbon nanotubes on clean substrates, Nano Lett. 9 (2009) 3137-3141.

[21] T. Wassmann, A. P. Seitsonen, A. M Saitta, M. Lazzeri, F. Mauri, Clar theory, $\pi$ electron distribution, and geometry of graphene nanoribbons, J. Am. Chem. Soc. 132 (2010) 3440-3451.

[22] D. Ye, H. Zhang, Extremal fullerene graphs with the maximum Clar number, Discr. Appl. Math. 157 (2009) 123-133.

[23] H. Zhang, D. Ye, An upper bound for the Clar number of fullerene graphs, J. Math. Chem. 41 (2007) 123-133. 